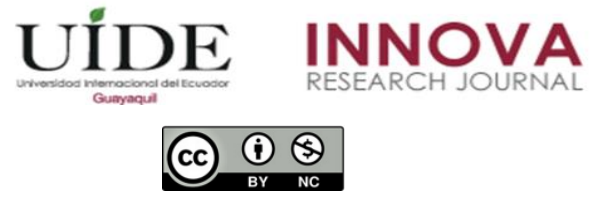

INNOVA Research Journal, ISSN 2477-9024

(Septiembre-Diciembre 2019). Vol. 4, No.3.2 pp. 131-143

DOI: https://doi.org/10.33890/innova.v4.n3.2.2019.1243

URL: http://revistas.uide.edu.ec/index.php/innova/index

Correo: innova@uide.edu.ec

\title{
Gestión comunitaria para la conservación de la Microcuenca Mónica, Loja, Ecuador
}

\section{Community management for the conservation of the Mónica Micro-basin, Loja, Ecuador}

Aurita Geovania Gonzaga Figueroa

Universidad Nacional de Loja, Ecuador

Autor por correspondencia: aurita.gonzaga@unl.edu.ec

Fecha de recepción: 21 de octubre del 2019 - Fecha de aceptación: 16 de diciembre del 2019

\section{Resumen}

El presente artículo propone la elaboración de un plan de manejo ambiental para la restauración y conservación de las riveras fluviales de la Microcuenca Mónica de Loja-Ecuador, mediante la fitorremediación. Esta microcuenca está siendo fuertemente afectada por la acción antrópica, el pastoreo incontrolado, la extracción de áridos, la expansión de viviendas próximas a los cauces, ampliación de la frontera agrícola y ganadera, descargas de efluentes de distintas actividades: domésticas, basurales, agrícolas y ganaderas. La metodología utilizada comprende actividades de campo y laboratorio, como: diagnóstico ambiental actual y análisis de muestras de agua. Identificados los contaminantes y basados en resultados del laboratorio se elaboró una propuesta de plan de manejo ambiental orientado a la gestión de manejo, restauración y conservación de las riveras fluviales. Las plantas utilizadas como fitorremediadoras son: Alnus acuminata Kunth (Aliso), Salix humboldtiana Willd (Sauce). Solanum torvum, Hedyosmum scabrum (Ruiz \& Pavón) y Medicago sativa (alfalfa), son especies nativas que se desarrollan en condiciones de humedad. Los resultados de esta investigación serán de gran utilidad para futuros proyectos que permitan la recuperación de estos ecosistemas para garantizar el desarrollo de procesos de recuperación y el manejo sustentable de las microcuencas.

Palabras claves: gestión comunitaria; plan de manejo ambiental; microcuencas

\begin{abstract}
This article proposes an environmental management plan for the restoration and conservation of the fluvial banks of the Mónica micro-basin in Loja-Ecuador, through phytoremediation. This micro-basin is being strongly affected by anthropic action, uncontrolled grazing, the extraction of aggregates, the expansion of housing near the channels, expansion of the agricultural and livestock border, discharges of effluents from different activities: domestic, garbage, agricultural and livestock This work includes a set of field and laboratory activities, such as: current environmental diagnosis of the microbasin to determine causes of contamination of the water resource; taking of water samples under technical criteria to avoid alterations of them. Once the main contaminants were identified and based on laboratory results, a proposal for an environmental management plan was drawn up, aimed at the management, restoration and conservation of the riverbanks, water treatment and environmental education as a support base. The plants that will be used for
\end{abstract}


phytoremediation were selected from the site since they are present in the site gives us an indication that they serve as phytoremediators: Alnus acuminata Kunth (Alder), Salix humboldtiana Willd (Willow). Solanum torvum, Hedyosmum scabrum (Ruiz \& Pavón) are species that develop in humid conditions. The results of this research will be very useful for future projects that allow the active or assisted recovery of these ecosystems and guarantee the development of recovery processes and the sustainable management of the micro-watersheds.

Key words: community management; environmental management plan; micro-watersheds

\section{Introducción}

La región Andina presenta una elevada diversidad de ecosistema resultantes de la interacción de procesos biofísicos a escalas continentales, subregionales y locales (CONDENSAN et al., 2016). Actualmente en estos ecosistemas se muestra una constante degradación por las diferentes presiones o modificaciones antropogénicas relacionadas con cambios en la cobertura vegetal (Murtinho et al., 2013), el incremento progresivo de la población, el aumento de la explotación de recursos (Machado et al., 2010) y la contaminación de las aguas (Acosta et al., 2009), provocando alteraciones a los elementos particulares en su perfil longitudinal (Guevara, 2013).

La conservación de los ecosistemas de microcuencas andinas se ha convertido en un hecho de suma importancia en la actualidad, debido a que cuentan con un elevado valor ecológico (Celleri y Feyen, 2009), mantienen estable la biodiversidad y proporcionan servicios y bienes ambientales, tales como la degradación de desechos orgánicos, la formación de suelos y el control de la erosión, la captura y almacenamiento de carbono (Rodríguez et al., 2012), la regularización y provisión hídrica (Iñiguez et al., 2014) y la prevención de consecuencias ecológicas futuras (Sudhakar et al., 2016). Los ecosistemas andinos también ofrecen una serie de Servicios Ambientales Hidrológicos, tales como la regulación del ciclo hidrológico, los altos rendimientos hídricos, el mantenimiento de la calidad del agua y la recarga de acuíferos (Quintero, 2010).

La situación actual de la microcuenca Mónica, está siendo fuertemente afectada por la acción antrópica: el pastoreo incontrolado, la extracción de áridos, la expansión de áreas urbanas próximas a los cauces, la roturación con objetivos agrícolas, acumulación de basura en lugares muy cercanos a la ribera; entre estos desechos se encontró escombros de materiales de construcción (cemento, ladrillos, cal, empaste, arena, etc); y desechos sólidos productos de actividades domésticas.

Otro factor que influye en la contaminación de la microcuenca es el pastoreo de ganado a pocos metros del rio. Los desechos del ganado se encuentran en las riberas y en los días de lluvia se transporta y se filtra al suelo ocasionando que, por acción de la escorrentía, se filtren y puedan llegar hasta el sub suelo contaminando el sector acuífero.

Finalmente se encontró que las personas que viven en el sector a parte de dedicarse a la ganadería también se dedican a actividades agrícolas, siendo otro factor que influye en la contaminación, por el uso de agroquímicos para cuidar y combatir las plagas que pudiesen ocasionar perjuicios a sus productos. 
En la parte baja de la microcuenca Mónica los agricultores utilizan agroquímicos y los suelos son aprovechados indiscriminadamente con todo tipo de aplicaciones sin darles tiempo de recuperación y en el peor de los casos, contaminándolos e inutilizándolos por completo; el excesivo empleo y uso de pesticidas, fertilizantes, herbicidas o demás agroquímicos para elevar la producción deteriora lentamente el suelo, y por si fuera poco, la contaminación por el residuo que éstos dejan con sus envases incrementa aún más la problemática, representan el primer agente contaminante del suelo, no solo por afectarlo directamente, sino que además extermina tanto a la plaga como a otras especies, generando un desequilibrio ambiental, daños a la salud de los agricultores, destrucción de flora, fauna y de los recursos naturales disponibles.

Por ello se hace crucial buscar tecnologías limpias que ayuden a descontaminar estos suelos con ellos se intenta reflexionar sobre métodos de control que a largo y corto plazo no provoquen pérdidas naturales ni daños a la salud.

La fitoremediación puede definirse como una tecnología sustentable que se basa en el uso de plantas para reducir in situ la concentración o peligrosidad de contaminantes orgánicos e inorgánicos de suelos, sedimentos, agua, y aire, a partir de procesos bioquímicos realizados por las plantas y microorganismos asociados a su sistema de raíz que conducen a un conjunto de métodos para degradar, asimilar, metabolizar o detoxificar metales pesados, compuestos orgánicos, radioactivos y petroderivados, que tengan la capacidad fisiológica y bioquímica para absorber, retener, reducción, mineralización, volatilización, estabilización, degradar o transformar dichas sustancias a formas menos tóxicas.

\section{Metodología}

\section{a) Ubicación del sitio}

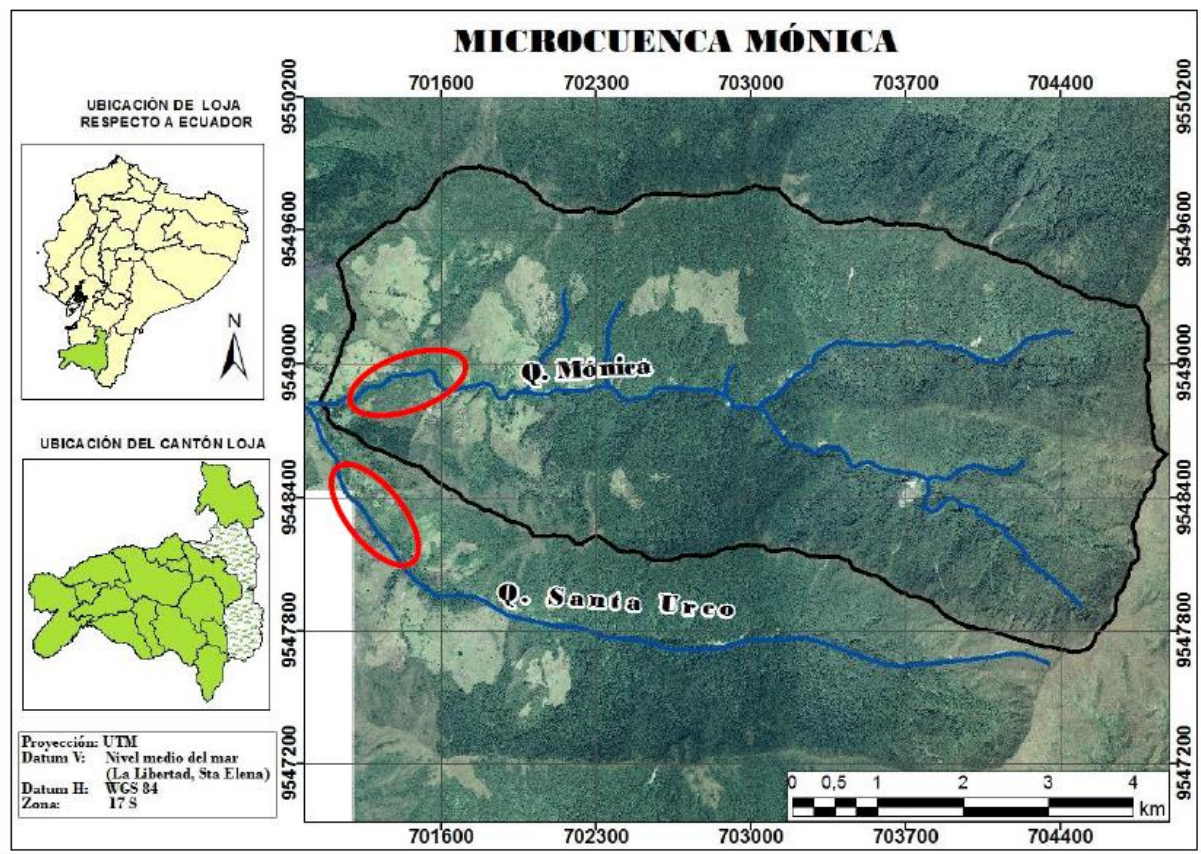

Figura 1. Ubicación del área de estudio, microcuenca Mónica 
La Microcuenca Mónica está ubicada en el cantón Loja en el sector conocido como "Dos Puentes", su rango altitudinal se distribuye entre las cotas que van desde los 2320 hasta los 3400 msnm (Vanegas, 2016). Según información temática disponible del MAGAP, escala 1:250 000 del año 2002, esta microcuenca cuenta con una superficie de $5.49 \mathrm{~km} 2$, con suelos de orden Inceptisol $(41,6 \%)$ y Entisol $(58,4 \%)$ que se define como los suelos que no muestran ningún desarrollo definido de perfiles no tiene "horizontes diagnósticos", y la mayoría es básicamente su material parental regolítico inalterado, y de capacidad tipo VII (aptas para explotación forestal) mayoritariamente.

\section{b) Características del área de estudio}

En lo que respecta a su vegetación se categoriza de la siguiente manera: bosque $71,2 \%$; pastizal 10,6\%; matorral 5,5\%; área sin vegetación 0,3\%; páramo 11,8\%; y plantaciones forestales $0,6 \%$. Y en cuanto a su condición climática, su temperatura oscila entre $10-14{ }^{\circ} \mathrm{C}$, con precipitaciones que fluctúan entre 1000 - $1250 \mathrm{~mm}$.

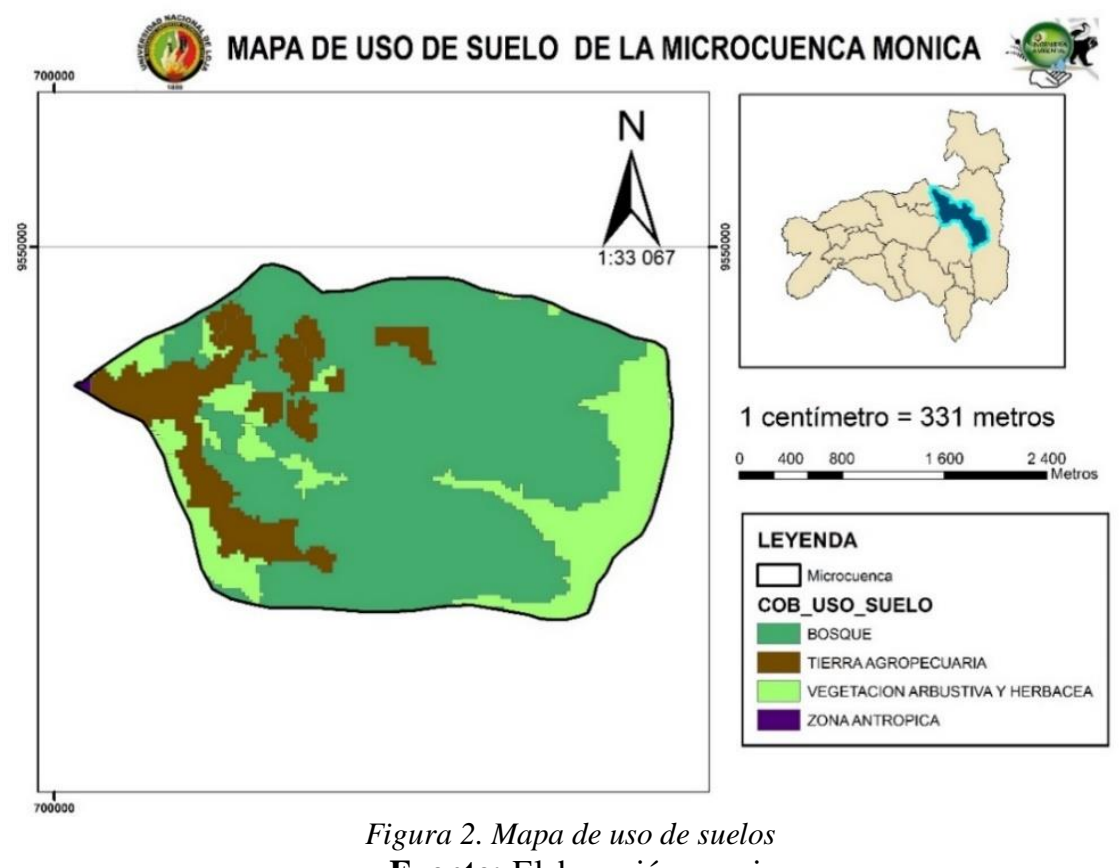

Fuente: Elaboración propia

El mapa nos indica el uso de suelo que tiene la parte baja de la microcuenca Mónica. Los porcentajes son similares entre el uso para actividades agropecuarias y la vegetación arbustiva y herbácea, lo que indica que es una zona no explotada en su totalidad teniendo una gran parte de terreno intangible. 


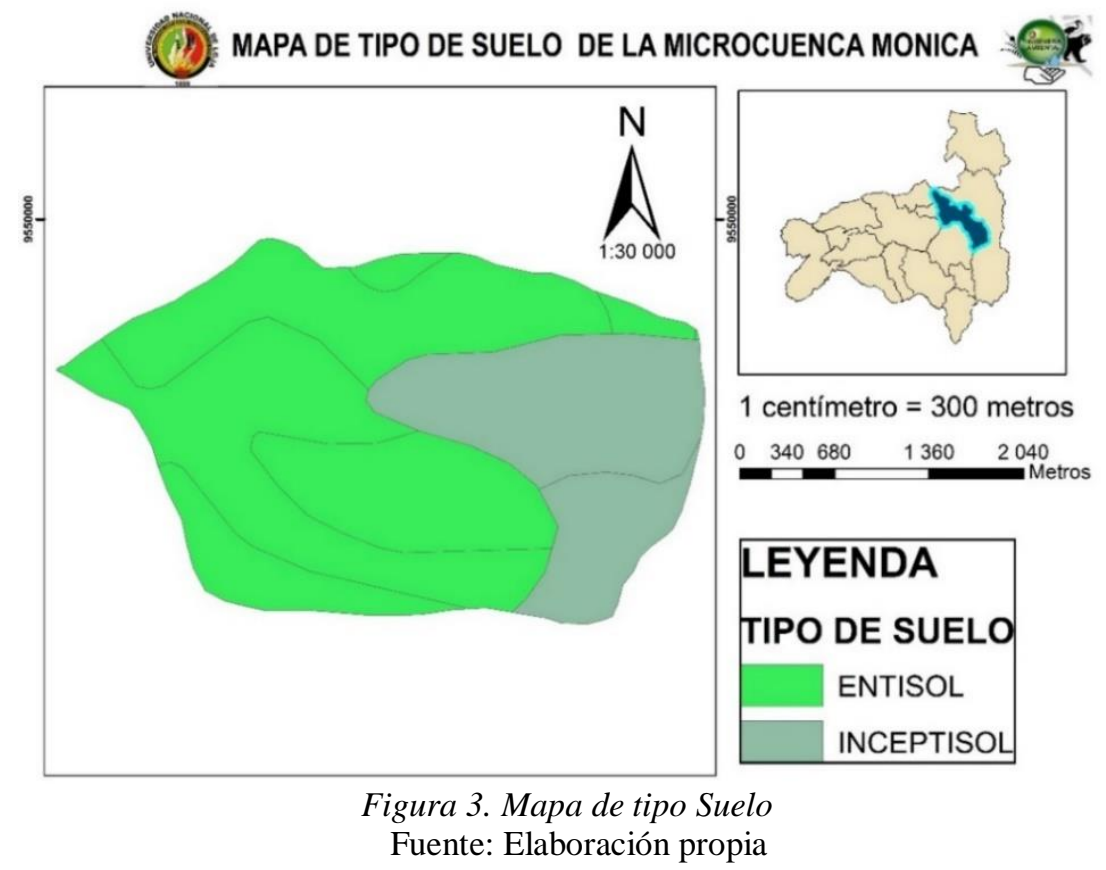

Materiales y Métodos

\section{a) Materiales}

\begin{tabular}{ll}
\hline En Campo & En Laboratorio \\
\hline Libreta de campo & Muestra de agua \\
GPS & Multiparametro \\
Ordenador & Turbidímetro \\
Recipiente receptor de muestra & Agua destilada \\
Fundas ziploc & \\
Cámara fotográfica & \\
Multiparametro & \\
Turbidímetro & \\
Agua destilada & \\
Entrevista & \\
\hline
\end{tabular}

\section{Diseño de la investigación}

Según Hernández et al. (1991) el diseño de investigación refiere al plan o estrategia concebida para obtener la información que se desea. Debido a este planteamiento, el diseño de la presente investigación es experimental - descriptiva. Es experimental, debido a que se pretende realizar y manipular las variables observando hechos reales y mediante estos realizar el análisis.

Con los antecedentes y diagnóstico de la situación actual del sitio se procedió a tomar muestras de agua y suelo para analizar su composición y determinar el nivel de contaminación que existe, y; de ser afirmativo, diseñar un plan de fitorremediacion que se pueda implementar en el sector para mitigar y controlar la contaminación de la microcuenca Mónica. 


\section{Resultados y Discusión}

Realización de muestreo y análisis IN SITU. Se realizaron 2 tomas de muestra de agua para medir turbidez y $\mathrm{pH}$.

Las muestras se recolectaron en la mañana, en la parte baja de la Microcuenca Mónica a $150 \mathrm{~m}$ de la entrada principal. Se hizo una medición in situ del pH y la turbidez, para luego trasladar las muestras al laboratorio de análisis de aguas en la Facultad Agropecuaria y de Recursos Naturales Renovables. Los datos in situ son los siguientes:

Tabla 1

Resultados de muestreo IN SITU

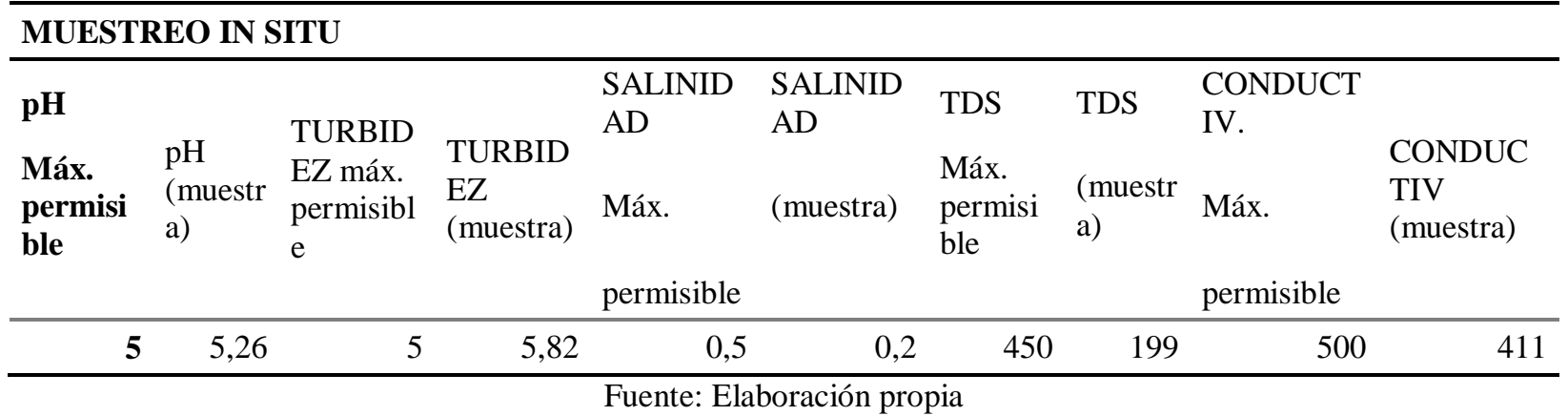

Según los datos registrados y comparados con la tabla de límites máximos permisibles referenciales de los parámetros de calidad de agua de la OMS, los valores que corresponden a $\mathrm{pH}$ sobrepasan de manera mínima al valor máximo establecido que es de 5 unidades de potencial de hidrogeno.

En cuanto respecta a la turbidez igualmente los valores sobrepasan mínimamente a lo establecido por los límites máximos permisibles que corresponde a 5 unidades de turbidez.

\section{Análisis de muestra EX SITU}

Las muestras de agua se llevaron al laboratorio, y reposaron por 24 horas en refrigeración, completado el periodo de tiempo indicado se procedió a realizar los análisis con los equipos especiales.

En la siguiente tabla se indican los valores obtenidos:

\section{Tabla 2}

Comparación de resultados IN SITU.

\begin{tabular}{llllll}
\hline MUESTREO IN SITU \\
\hline $\mathbf{p H}$ & TEMPERATURA & TURBIDEZ & CONDUCTIVIDAD & T.D.S & SALINIDAD \\
\hline $\mathbf{5 , 2 6}$ & $12,5^{\circ} \mathrm{C}$ & 5.82 & 411 & 199 & 0.2 \\
\hline \multicolumn{5}{c}{ Fuente: Elaboración propia } \\
\hline
\end{tabular}




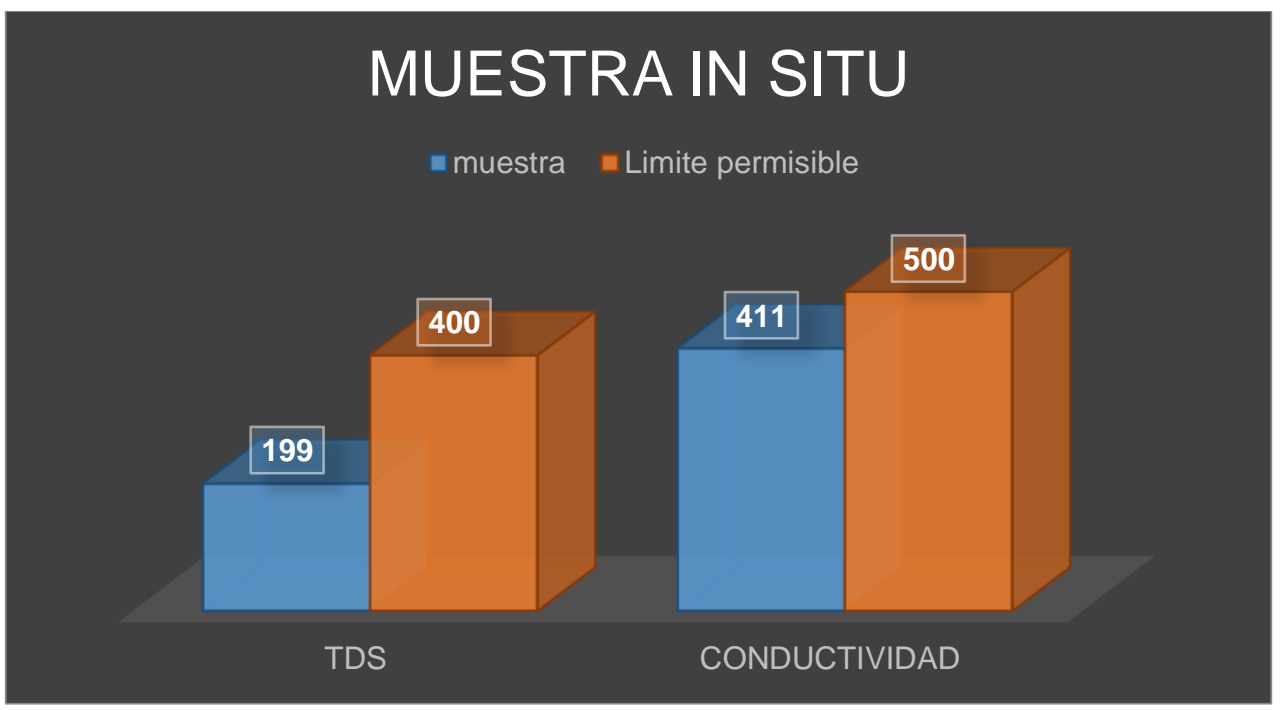

Tabla 3.

Resultados EX SITU.

\begin{tabular}{ll}
\hline \multicolumn{2}{l}{ MUESTREO EX SITU } \\
\hline pH / Temperatura & TURBIDEZ \\
$\mathbf{6 , 2 9} / \mathbf{3 . 5}^{\circ} \mathrm{C}$ & 8.7 \\
\hline
\end{tabular}

Fuente: Elaboración propia

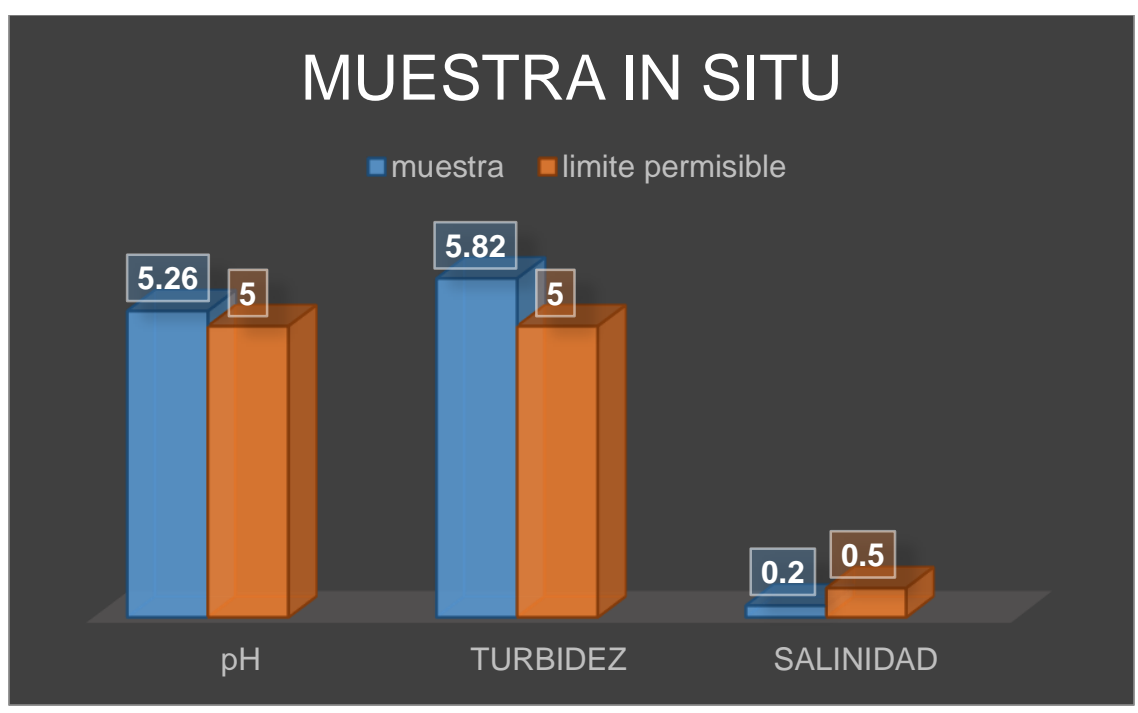

Los resultados del análisis EX SITU varían completamente de los resultados IN SITU por lo que se puede concluir anticipadamente que, el reposo de las muestras por 24 horas estabilizaron los valores teniendo un porcentaje más alto de fiabilidad.

En la siguiente tabla se puede observar de mejor manera la comparación de resultados obtenidos con los límites permisibles. 
Tabla 4.

Comparación de resultados EX SITU.

Fuente: Elaboración propia

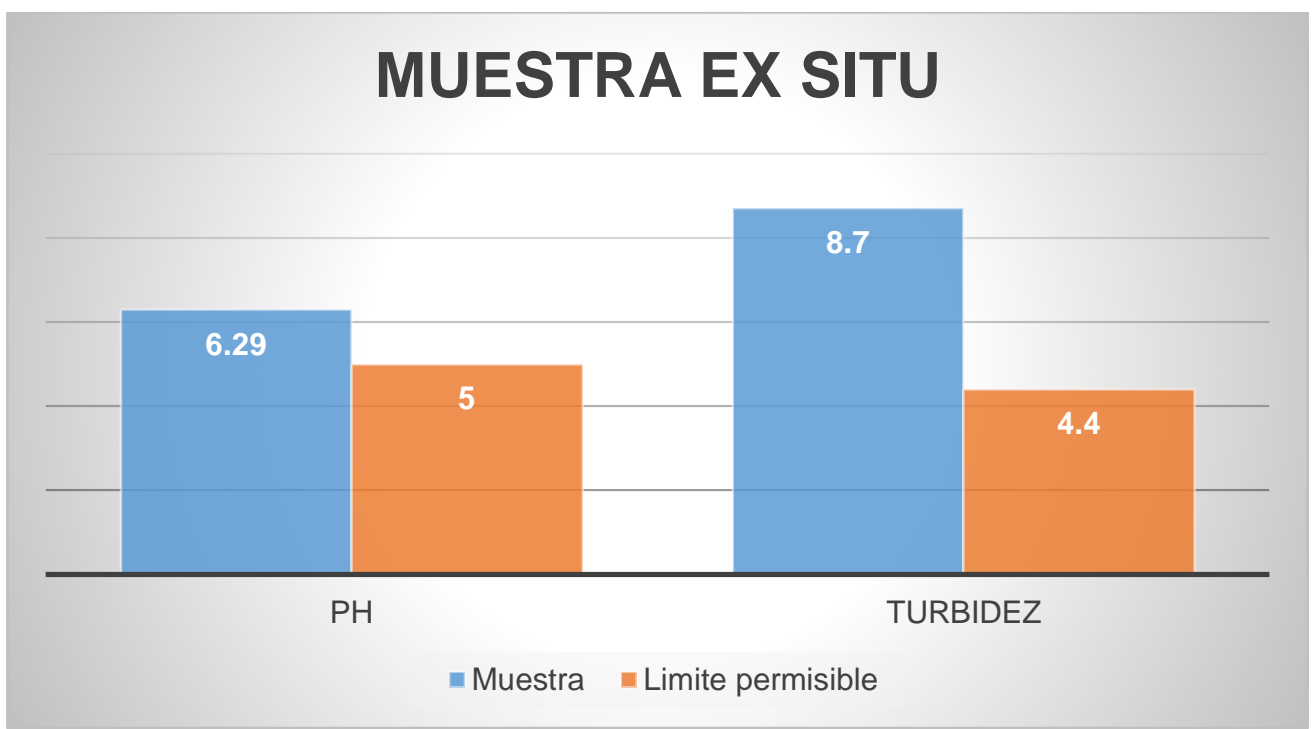

"Morocho (2016), señala que la microcuenca Mónica es considerada como una microcuenca degradada por que el $3 \%$ de la microcuenca se dedica pastizal debido a que la cubierta vegetal ha sido eliminada por la ganadería intensiva, el $4 \%$ destinado a plantaciones de pino y el 5\% matorral producto de las actividades antrópicas, mientras que el bosque ocupa $71 \%$ del área de la microcuenca." "El 48,76\% de la microcuenca se encuentra en Parque Nacional Podocarpus. Las microcuencas en la zona de amortiguamiento del PNP han sido muy afectadas por las actividades antrópicas, debido a la falta de manejo y conservación de las microcuencas (FORAGUA, 2013).”

De acuerdo a Miller et al, (2011) citado por Morocho (2016) indica que las fuentes alternativas fuera del río (bebederos) es otra opción ya que mejora la salud de la ribera por impedir que el ganado beba directamente del cuerpo hídrico y provoque la contaminación del mismo, también, mejora las características de la vegetación (cobertura del dosel, área basal total, mantillo, suelo desnudo) y las propiedades del suelo (suelo de NO3-N) cerca del río. La aplicación de esta técnica permite la regeneración de los bosques riparios, mejoran la calidad del agua y estabilizan los márgenes.

Esparza, (2017), indica en lo referente a la determinación de los parámetros físicoquímicos realizados en los ríos de la hoya de Loja, reflejan tener una buena calidad del agua, conforme a los límites permisible de la Norma de Calidad Ambiental. Sin embargo, los parámetros tienden a alterar sus concentraciones al existir presencia de actividades antrópicas en los alrededores, lo cual ha afectado el desarrollo de los macroinvertebrados. 
De acuerdo al estudio realizado, luego de los procesos anteriormente indicados se puede llegar a concluir que el agua que pasa por la parte baja de la Microcuenca Mónica sobrepasa los valores máximos permisibles establecidos por la OMS lo que indica que no es apta para actividades de consumo humano ya sea directamente o indirectamente (agricultura).

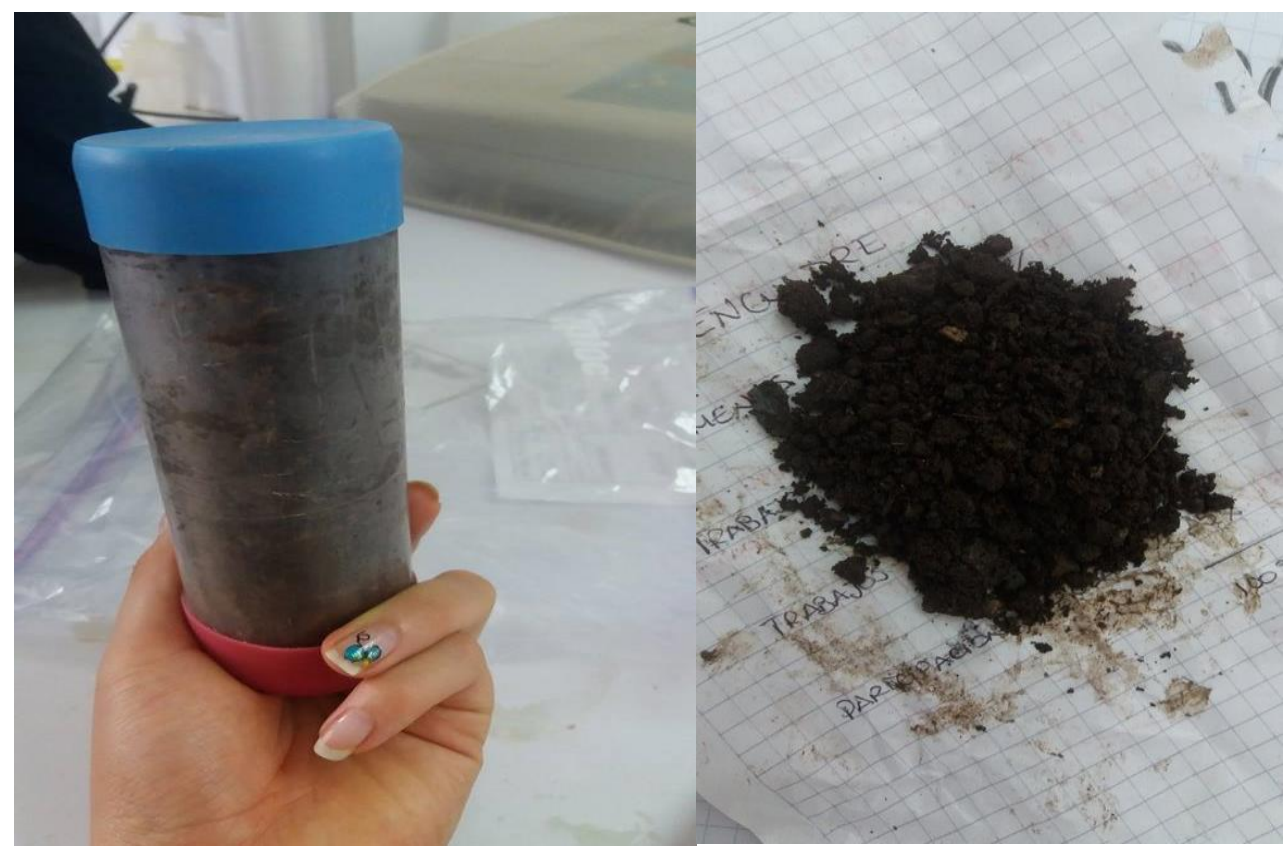

La técnica utilizada en el plan de manejo ambiental que se ha seleccionado de acuerdo con las necesidades de la investigación y principalmente con el objetivo de cumplir las expectativas previstas al inicio del proyecto, es la fitorremediación. Las ventajas que ofrece esta técnica, no solo es de carácter económico y social, sino por su elevada eficiencia al momento de capturar, extraer y minimizar los contaminantes presentes en el suelo, sobrepasan las ventajas que ofrecen otros tratamientos.

La especie de planta que se utilizará en nuestro proyecto serán: Alnus acuminata Kunth (Aliso), Salix humboldtiana Willd (Sauce). Solanum torvum, Hedyosmum scabrum (Ruiz \& Pavón), y especies de plantas herbáceas como la alfalfa cuyo nombre científico es Medicago sativa, pertenecientes a las familias de las Fabáceas o leguminosas. Ya que sus raíces pueden llegar a los 4 metros, son especializadas en absorber gran cantidad de humedad y minerales y entre otros componentes también absorben contaminantes, ayudando a mejorar las condiciones físicas y químicas del suelo. Además se caracteriza por fijar nitrógeno y fósforo en el suelo, son tolerantes a bajas temperaturas, entre 10 y $15^{\circ} \mathrm{C}$ bajo cero y temperaturas medias anuales de $15^{\circ} \mathrm{C}$, Rizoremediación, por la Pseudomona fluorescens, regula mediante su sistema raíces de la alfalfa; además esta plata tiene la capacidad de albergar gran número de bacterias en sus sistemas radiculares, las mismas que ayudan a degradar los compuestos orgánicos no obstante es una planta tolerante a la sequía pero muy susceptible a excesos de agua y a suelos ácidos por debajo de un $\mathrm{pH}$ de 6,4 . 


\section{Aplicación del plan}

La metodología propuesta, recoge todas las etapas necesarias para lograr una selección o diseño adecuado, en función de las condiciones particulares del suelo y las características de los contaminantes que contiene.

La siembra de las plantas utilizadas como fitorremediadoras, se realizará en los bordes de las riveras de la microcuenca de manera directa en el terreno libre de vegetación (Aliso, Sauce, Faique, Solanum, alfalfa); y se empezará con el cultivo ex situ de semillas de alfalfa (semilleros), para asegurar el nacimiento y crecimiento óptimo de la planta.

Luego del periodo previo al cultivo, se trasladará las plántulas hacia el terreno seleccionado para proceder a realizar la fase in situ, que comprende el crecimiento total de la planta y su posterior extracción con los contaminantes contenidos en sus raíces.

Para este proceso es muy importante el monitoreo y evaluación constante, para comprobar la eficiencia de la técnica, el estado de la planta y su adaptación al suelo, y por último el comportamiento de la planta frente al contaminante (adaptación o rechazo).

\section{Evaluación del plan}

Para el proceso de evaluación se realizarán visitas programadas en diferentes horarios para registrar el crecimiento y desarrollo normal de las plantas. Una parte fundamental de este proceso será extraer muestras representativas del suelo para trasladarlas al laboratorio y analizar las características físicas y químicas y determinar si se ha generado un cambio sustancial en el terreno contaminado por agroquímicos.

Otro componente a evaluar serán diferentes partes de las plantas sembradas en el sitio como raíces, tallo, hojas. El análisis se realizará ex situ, puntualmente en el laboratorio para determinar si las especies sembradas están cumpliendo su objetivo y está extrayendo las cantidades de agroquímico esperadas.

\section{Conclusiones}

Es importante en la primera etapa realizar un diagnóstico general del suelo contaminado. Se ha podido comprobar dicha contaminación por factores externos, como el vertido de basura al sector en zonas cercanas a la ribera. Esta basura es de diferentes tipos, desde desechos comunes de procedencia doméstica hasta escombros provenientes de residuos de materiales de construcción.

Una vez caracterizado el suelo a tratar, se pasa a seleccionar y evaluar las propuestas de tecnologías a desarrollar a escala de laboratorio, siempre es recomendable valorar un proceso independiente de otro, al concluir el estudio, realizar el análisis de secuencias de tratamientos, conocido como tren de tratamientos. 
En la parte baja de la microcuenca Mónica el uso de insecticidas químicos sustenta la actividad agrícola, misma que ha generado problemas de contaminación en el suelo y agua, esto a través de las descargas de estas sustancias a los sistemas lagunares, vía riego y arrastre por la lluvia.

Considero que la técnica más óptima es la Fitorremediacion por sus dos ventajas más llamativas. La primera: es el bajo costo que se emplea para poner en marcha esta técnica; y la segunda: los excelentes resultados que se ha demostrado como una técnica estabilizadora de los biocomponentes del agua y suelo en presencia de contaminantes.

Con la técnica de fitoremediación planteada se pretende mitigar el suelo contaminado por agroquímicos en la zona baja de la microcuenca Mónica ubicada en el sector dos puentes de la ciudad de Loja y de esta manera se devuelve a la microcuenca su estado de naturalidad y paisajismo que ha sido degradado durante los últimos años.

Se concluye que mediante la fitoremediación con plantas arbustivas propias del lugar (Aliso, Sauces, Faiques, Mortiños) y con el uso de la especie Medicago sativa se lograra descontaminar el suelo de la zona baja de la microcuenca Mónica ubicada en el sector dos puentes de la ciudad de Loja. Mediante las propiedades físicas y químicas del suelo y agua se estableció que la especie Medicago sativa es apta para este tipo de suelos.

Es muy importante establecer estrategias de restauración de las franjas ribereñas en la microcuenca Mónica a través de la fitorremediación las mimas que están enfocadas a la conservación y prevención de la alteración de las funciones hidrológicas.

\section{Bibliografía}

Acosta, C. (2009). Estudio de la Cuenca Altoandina del río Cañete (Perú): Distribución altitudinal de la comunidad de macroinvertebrados bentónicos y caracterización hidroquímica de sus cabeceras cársticas. Ecología. Universidad de Barcelona.

Acosta, R., Ríos, B., Rieradevall, M., y Prat, N. (2009). Propuesta de un protocolo de evaluación de la calidad ecológica de ríos andinos (CERA) y su aplicación a dos cuencas en Ecuador y Perú. Limnetica, 28(1), 35-64.

Budhu, M. (2006). Soil mechanics and foundations.

Carter, R. (2002). Soil quality for sustainable land management: organic matter and aggregation interactions that maintain soil functions.

Cartografia.cl. (2012). cartografia.cl. Recuperado el 21 de 11 de 2016, de http://www.cartografia.cl/beta/index.php/home/percepcion/830-2013-08-21-02-17-46

Cando Rodríguez, Miguel Angel. (2012). Determinación y análisis de un proceso de biorremediación de suelos contaminados por hidrocarburos. Cuenca: Universidad Politécnica Salesiana

Celleri, R., y Feyen, J. (2009). The Hydrology of Tropical Andean Ecosystems: Importance, Knowledge Status, and Perspectives. International Mountain Society, 29(4), 350-355.

Chacón Mejía, N. (2012). Topografia Elemental. Loja: UTPL.

Civallero, E. (Julio de 2010). Tierra de vientos. Obtenido de http://tierradevientos.blogspot.com/2010/07/cultura-andina.html 
Codenpe. (Enero de 2014). CODENPE. Obtenido de http://www.codenpe.gob.ec/index.php?option=com_content $\&$ view=article\&id=158:sara guro\&catid $=85$

Conaf. (2015). Reporte Especial de Actividad Volcánica. Santiago.

CONDENSAN. 2016. Consorcio para el desarrollo Sostenible de la Ecorregion Andina

Direccion Nacional de Cartografia y Medio Ambiente. (2012). inegi.org.mx. Recuperado el 21 de 11 de 2016, de http://www.inegi.org.mx/geo/contenidos/imgpercepcion/imgsatelite/doc/aspectos_tecnic os_imagenes_landsat.pdf

Doran, M. (1994). Defining soil quality for a sustainable environment. .

El Clarin. (25 de Julio de 2017). Smog en China. Clarin Mundo, pág. 5.

Estrella, J., Manosalvas, R., Mariaca, J., \& Ribadeneria, M. (2005). Biodiversidad y recursos genéticos: una guía para su uso y acceso en el Ecuador. En J. Estrella, R. Manosalvas, J. Mariaca, \& M. Ribadeneria, Biodiversidad y recursos genéticos: una guía para su uso y acceso en el Ecuador (pág. 31). Quito: Ediciones Abya Yala.

Esparza Godoy, Karina Rebeca, (2017). Influencia del estado de conservación de las microcuencas andinas sobre la diversidad de macroinvertebrados acuáticos. Tesis de grado, Carrera Manejo y Conservación del medio Ambiente, Facultad Agropecuaria y de Recursos Naturales Renovables. Universidad Nacional de Loja.

Fuentes, J. D. (2004). Análisis morfométrico de cuencas. Caso de estudio del Parque Nacional Pico de Tancpitaro. Instituto Nacional de Ecologia.

Guevara, G. (2013). Evaluación ambiental estratégica para cuencas prioritarias de los Andes colombianos: dilemas, desafíos y necesidades. Acta Biológica Colombiana, 19(1), 11-24

Grupo Salinas. (Marzo de 2016). Salinerito. Obtenido de http://www.salinerito.com/economiasolidaria/que-es-la-economia-solidaria

Iñiguez, C., Leiva, A., Frede, H.-G., Hampel, H., y Breuer, L. (2014). Deforestation and benthic indicators: how much vegetation cover is needed to sustain healthy Andean streams?, 9(8), 10.

Iñiguez, C., Rausche, S., Cueva, A., Sánchez, A., Espinosa, C., y Breuer, L. (2016). Shifts in leaf litter breakdown along a forest-pasture-urban gradient in Andean streams. Ecology and Evolution, 6(14), 4849-4865.

Jácome, W. (2013). Revista Líderes. Obtenido de http://www.revistalideres.ec/lideres/salineritoejemplo-economia-solidaria.html

Machado, D., Pineda, M. C., Viloria, J., y Casanova, E. (2010). Evaluación ambiental , agrícola y forestal de tierras de la cuenca alta del rio Guarico, Venezuela. Venesuelos, 49-60.

Masera, L. (1999). Sustentabilidad y manejo de recursos naturales.

Mena, P. (2011). La Biodiversidad del Ecuador. Quito.

Ministerio de Agricultura de Chile. (2016). Incendios Forestales en Chile. Santiago.

Montenegro Gallardo, Fabián. Aislamiento y selección de cepas bacterianas nativas de suelos de la xii región de chile, para la degradación de crudos de petróleo. [En línea] 2007. http://cybertesis.uach.cl/tesis/uach/2007/fam777a/doc/fam777a.pdf.

Morocho, Fanny. (2016). "Evaluación de la calidad del bosque ripario en cuencas prioritarias del Cantón Loja", Tesis de grado, Carrera de Ingeniería en Manejo y Conservación del Medio Ambiente, Facultad Agropecuaria y de Recursos Naturales Renovables Universidad Nacional de Loja. 
Murtinho, F., Eakin, H., López-Carr, D., y Hayes, T. M. (2013). Does external funding help adaptation? Evidence from community-based water management in the Colombian Andes. Environmental Management, 52(5), 1103-1114.

Orosco Verdezoto, Verónica Paulina. biorremediación de vegetación contaminada con petróleo por derrames en el campamento guarumo - petroproducción. Tesis de grado, Riobamba: Escuela Superior Politécnica de Chimborazo, Facultad de Ciencias, Escuela de Ciencias Químicas, 2008.

Ortiz Bernad, Irene, y otros. Informe de Vigilancia Tecnologica. [En línea] http://www.madrimasd.org/informacionidi/biblioteca/publicacion/doc/vt/vt6_tecnicas_r ecuperacion_suelos_contaminados.pdf

Paretas, J. (2006). Regionalización de gramíneas, leguminosas y árboles multipropósitos.

Santamaría, J., \& Sanz, T. (2005). Manual de practicas de Topografia y Cartografia. Madrid.

Sparlyng, G. (1997). Soil microbial biomass, activity and nutrient cycling, as indicators of soil health. CAB.

Samanez Gibaja, Elizabet. Biodegradación bacteriana por bioestimulación en suelos contaminados con petróleo crudo. [En línea] facultad de farmacia y bioquimica unidad de post grado; universidad macional may de san marcos, 2008. http://www.cybertesis.edu.pe/sisbib/2008/samanez_ge/pdf/samanez_ge.pdf.

Schmidt, Wini. Suelos contaminados con hidrocarburos. [En línea] http://www.ingenieroambiental.com/3021/Bioremed_Mex2.pdf. 2011, Journal of Hazardous Materials, pág. 10

Sudhakar, C., Manaswini, G., Satish, K., Singh, S., Jha, C., y Dadhwal, V. (2016). Conservation priorities of forest ecosystems: Evaluation of deforestation and degradation hotspots using geospatial. Ingeniería Ecológica, 91, 333-342.

Torres Delgado, Katerine. Biorremediación de suelos contaminados por hidrocarburos. Medellín: Universidad Nacional de Colombia, Facultad de Minas, Ingeniería Química, 2009. 